Review

Article

\title{
Evidence and Challenges in Left Atrial Appendage Management
}

Taira Yamamoto, MD, PhD, Daisuke Endo, MD, PhD, Satoshi Matsushita, MD, PhD, Akie Shimada, MD, PhD, Keisuke Nakanishi, MD, PhD, Tohru Asai, MD, PhD, and Atsushi Amano, MD, PhD

\begin{abstract}
This review aimed to discuss the anatomical properties of the left atrial appendage (LAA), its relationship with atrial fibrillation (AF), effectiveness of LAA occlusion (LAAO), techniques, and new devices used to perform this procedure. An electronic search was performed to identify studies, in the English language, on LAA management. Searches were performed on PubMed Central, Scopus, and Medline from the dates of database inception to February 2020. For the assessed papers, data were extracted from the reviewed text, tables, and figures, by two independent authors. Anticoagulant therapy for patients with AF has proven beneficial and is highly recommended, but it is challenging for many patients to maintain optimal treatment. Surgery is the most cost-effective option; surgical methods include simple LAA resection, thoracoscopic surgery, and catheter treatment. Each procedure has its advantages and disadvantages, and many prospective studies have been conducted to evaluate various treatment methods. In managing the LAA, dissection of the LAA, such as changes in its shape and size due to remodeling during $\mathrm{AF}$, changes in autonomic nerve function, and thrombosis, must be understood anatomically and physiologically. We believe that early treatment intervention for the LAA should be considered particularly in cases of recurrent AF.
\end{abstract}

Keywords: atrial fibrillation, stroke, left atrial appendage, cardiac surgery, endocrine function

\section{Introduction}

Atrial fibrillation (AF) is a type of tachyarrhythmia that increases in frequency with age and is often

Department of Cardiovascular Surgery, Juntendo University, Tokyo, Japan

Received: February 19, 2021; Accepted: May 11, 2021

Corresponding author: Taira Yamamoto, MD, PhD. Department of Cardiovascular Surgery, Juntendo University, 2-1-1 Hongo Bunkyo-ku, Tokyo 113-8421, Japan

Email: tyamamo@juntendo.ac.jp

${ }^{1}$ This is a secondary publication of the paper published in "Japanese Journal of Cardiovascular Surgery", Vol.50, No.1, pp. xxxvi xlviii, January 2021.

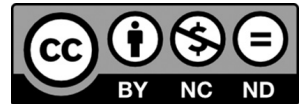

This work is licensed under a Creative Commons Attribution-NonCommercialNoDerivatives International License.

(C)2021 The Editorial Committee of Annals of Thoracic and Cardiovascular Surgery diagnosed in general clinical practice. The frequency of complications of cardiogenic stroke increases with chronic AF. In the cases of AF, the left atrial appendage (LAA) is the most critical site where thrombi occur. Therefore, management of the LAA is essential for preventing cerebral infarction. Here, we discuss the anatomical properties of the LAA, its relationship with AF, effectiveness of LAA occlusion (LAAO), techniques, new devices used to perform this procedure, and their cost-effectiveness.

\section{Anatomical Characteristics of the Left Atrium and LAA}

It is necessary to comprehend the anatomical and histological characteristics of the atria, atrial appendage, and pulmonary vein (PV) from an embryological perspective; hence, we considered a study conducted by 
Yamamoto T, et al.

Dr. Tsukasa Saito. ${ }^{1)}$ During the fetal stage, atrial structures such as the orifices of the superior and inferior vena cava, the coronary sinus, the PV, the foramen ovale, the left and right halves of the heart, and crista terminalis are formed, and many of these structures develop anatomical barriers contributing to re-entry. Simultaneously, structures such as the isthmus between the inferior vena cava and the tricuspid valve, the cavotricuspid isthmus, and the left mitral isthmus are also formed. These structures play an important role in re-entry and serve as targets for electrocautery procedures. Moreover, a major difference between the atria and the ventricles is that the atrial muscles have a complex three-dimensional structure, and the cell composition and distribution in the atria are more diverse.

The process by which the LA is formed differs greatly from that of the ventricles. During the fetal stage of development, the left umbilical and left vitelline veins disappear, and the left common cardinal vein gradually narrows and forms Marshall's vein, which eventually becomes Marshall's ligament. The left horn of the sinus venosus, which splits from the left side of the atrium to form the coronary sinus, and the right umbilical vein, which empties into the right horn, also disappears. The remaining right vitelline vein and the right common cardinal vein are the origins of the inferior and superior vena cava, respectively. Once the primitive atrium fuses with the right horn of the sinus venosus and enlarges, the atrial septum tissue develops to form the LA. Around the gestational age of 5 weeks, the fetal body is between 5 and $6 \mathrm{~mm}$ long. At this stage of development, the fetal PV gradually appears as a sac-like protuberance on the left side of the atrial septum, in a superoposterior direction to the primitive atrium. This protuberance extends toward the visceral venous plexus at the lung bud (lung anlage) during development and forms the common PV (Fig. 1A). At the gestational age of 6 weeks, the common PV is gradually absorbed from the medial side to the LA. Then, the peripheral left and right PVs are taken in, and each of these links is directed to the LA. Once the fetus achieves a gestational age of 8 weeks, the branches of the left and right PVs are inserted into the LA, and the four PVs form orifices in the LA (Fig. 1B). The posterior wall of the LA is formed through this process of vessel insertion, and as a result, from tan embryological perspective, has its origins in the PVs. Structures equivalent to the right and left atria during the fetal stage remain as the right and left appendages, which maintain a characteristic columnar structure.

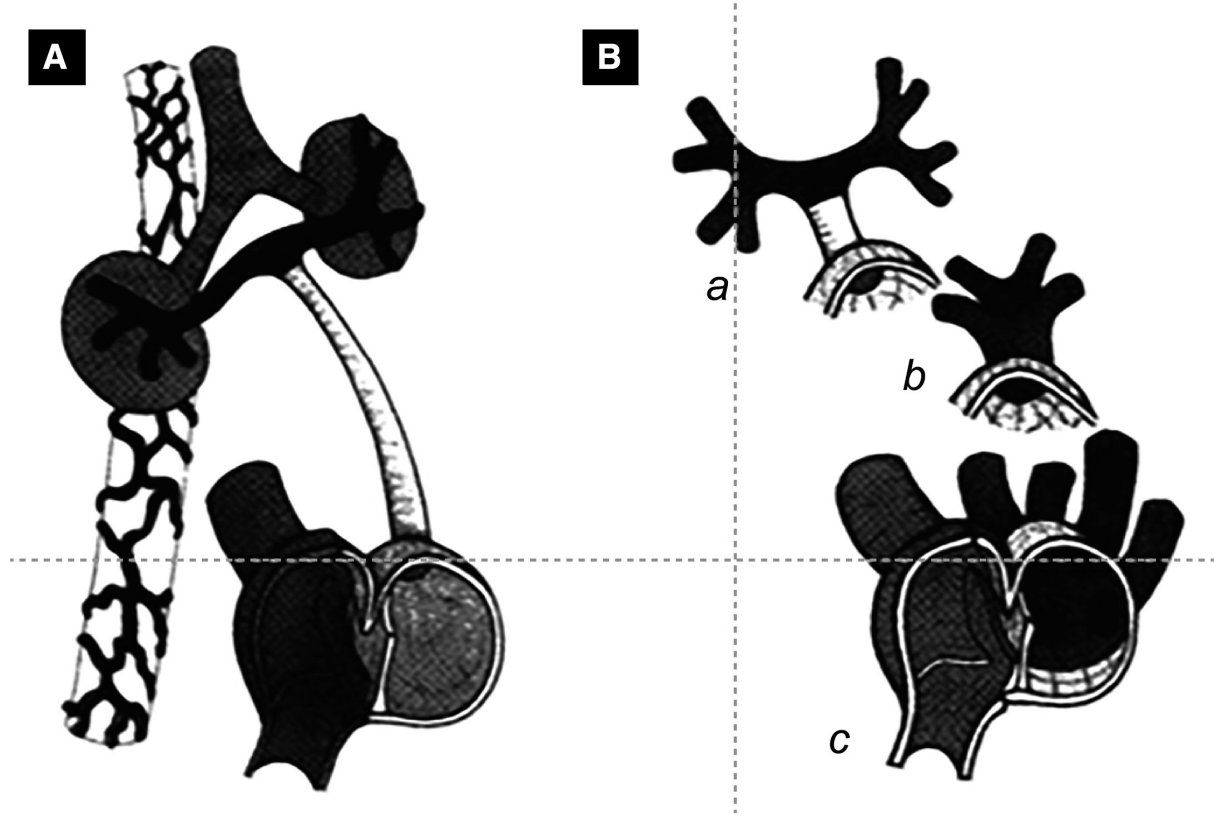

Fig. 1 (A) At this stage of fetal development, the fetal PV gradually appears as a sac-like protuberance on the left side of the atrial septum, in a superoposterior direction of the primitive atrium. This protuberance extends toward the visceral venous plexus at the lung bud (lung anlage) during development and forms the common PV. (B-a) In the later stages of development, the common PV is gradually absorbed from the medial side to the LA. (B-b) Then, the peripheral left and right PVs are also placed in, and each of these links is directed to the left atrium. (B-c) Eventually, the branches of the left and right PVs insert into the LA, and the four PVs form orifices in the LA. ${ }^{1)}$ LA: left atrium; PV: pulmonary vein 


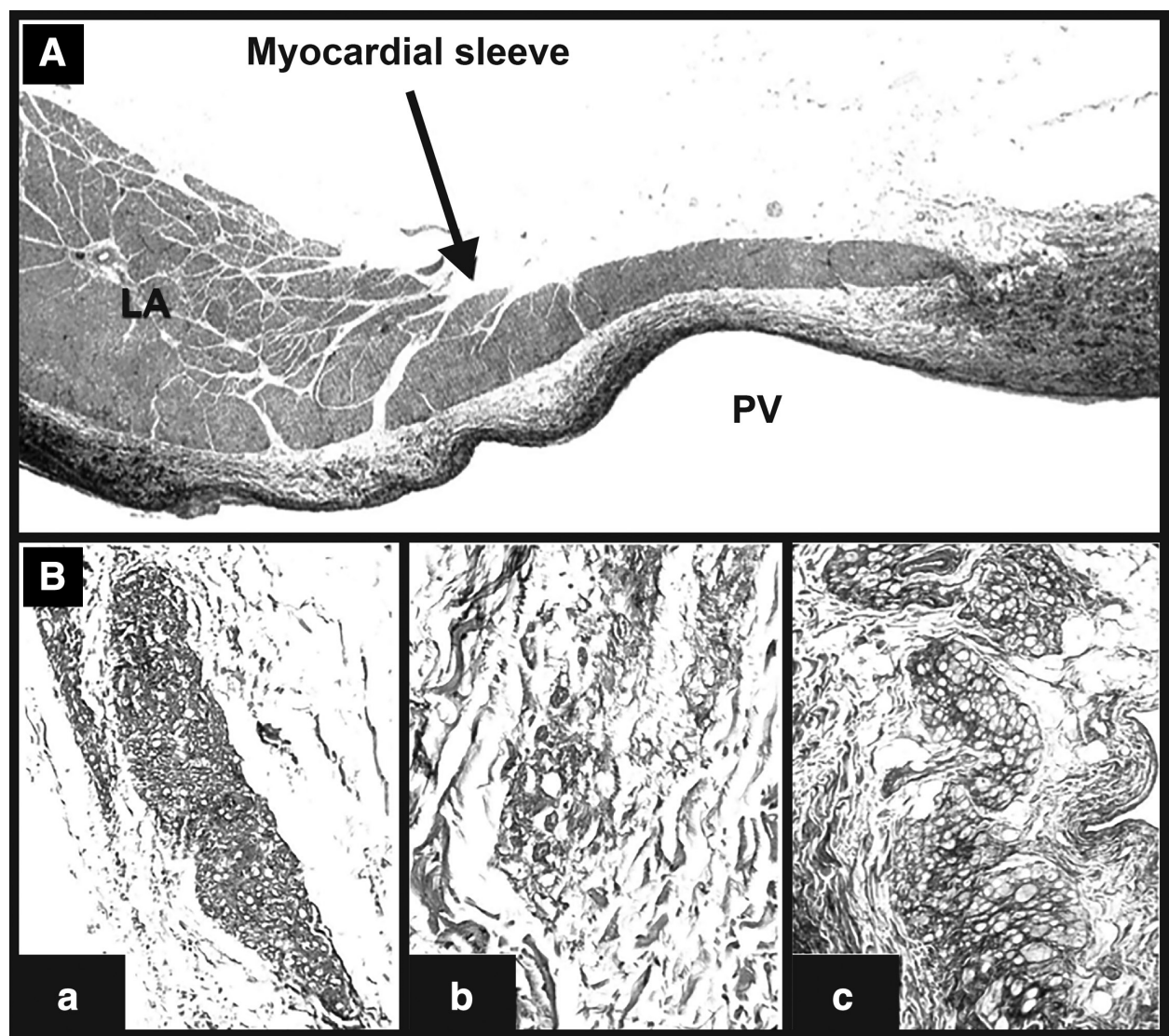

Fig. 2 (A) The atrial wall gradually thins toward the hilar bundle. The atrial muscle layer and the medial smooth muscle layer of the PV do not fuse with each other. (B) Histological image of the atrial muscle around the PV; (a) on the PV's peripheral side, small cells with fibrosis form a small population in an island shape (Azanmallory staining X20); (b) small cells can be seen individually isolated (Azanmallory staining X40); (c) atrial muscle swelling with fibrosis (Azan-Mallory staining X10). ${ }^{1)}$ LA: left atrial muscle; PV: pulmonary vein

\section{Characteristics of the Atrial Muscles Sur- rounding the PV (Fig. 2)}

Careful observation of the atrial muscles extending into the area surrounding the PV revealed gradual thinning with extension toward the hilum of the lungs. The atrial muscle layers are separated by the medial smooth muscle and fibro-adipose tissue of the PV, located on the external side without merging from the orifice of the PV to the peripheral end of the hilum of the lung. The electric potential found within the PV is not due to the medial smooth muscle, but rather originates in the atrial muscle on the external side. The PV stricture, which is problematic in ablation procedures performed from the vascular lumen in the cases of AF, is caused by contractile remodeling of the vascular wall. Thus, it is necessary to have a comprehensive understanding of the anatomical characteristics of the atrial muscle, which is the target of ablation, as it is located on the external side of the PV wall.
As the myocardial cells approach the peripheral end of the PV (the hilum side), they become smaller and fibrous, and adipose invasion often occurs in the interstitial region, exhibiting an increasing diversity of tissues. Histological observation of the atrial muscle sleeve in AF reveals the opening of spaces between the muscle bundles as a result of increased collagen fibers; thus, the remaining atrial muscle also presents a variety of findings such as enlargement and degeneration. This type of tissue (anatomical) remodeling results in increased fluctuations in the refractory period, transmission speed, and automaticity at the affected site. This, in turn, promotes electrical remodeling and arrhythmogenic responses.

\section{Morphological Classification of the LAA}

The LAA arises from the primitive atrium and is formed by the adsorption of the PV and its branches. ${ }^{1,2)}$ 
The LAA is clearly suspended from the LA. It is a cylindrical sac (diameter: 10-20 mm, the thickness of inner column: $1-2 \mathrm{~mm}$ ) that bends slightly to the right, adopting the form of a chicken wing and extending toward the anterior wall of the LA in the vicinity of the pulmonary artery. The orifice of the LAA lumen is either elliptical or round. Although the wall of the LA is smooth, the inner surface of the LAA develops in a columnar form. The left PV is located at the back of the entrance to the LAA, and the entrances to both the LAA and the left PV are divided by a ridge. The LAA is long with a narrow neck; thus, there is a high risk of blood stasis within the structure when the patient experiences arrhythmia. According to the studies classifying the LAA according to morphology types using computed tomography (CT), the most common type was the chicken wing (48\%), followed by the cactus (30\%), windsock (19\%), and cauliflower (3\%) (Fig. 3). This classification considered the cactus, windsock, and cauliflower as the "non-chicken wing" types in which cardiogenic cerebral embolism events occurred frequently. In particular, the cauliflower type was most commonly associated with cardiogenic cerebral embolism events because there was an eightfold chance of embolism compared to the chicken wing type. ${ }^{3)}$

\section{Physiological Functions of the LAA}

\section{Endocrine function}

The atrium is a rich source of atrial natriuretic peptide (ANP), which regulates the balance between salt and bodily fluids. Specialized endothelium cells involved in the production and release of natriuretic peptides exist in both the LA and LAA. In particular, the LAA is the main site of ANP production in the heart, and $\sim 30 \%$ of the ANP synthesized by the heart is stored in the granulocytes. The ANP concentration in the left atrial wall is $\sim 40$ times that contained in the right atrium and ventricles. ${ }^{4,5)}$ This function enables the heart to adapt to sudden changes in cardiac capacity, for example, during heart failure.

\section{Reservoir function}

The LAA has a reservoir function because it is the most expandable structure in LA. It also functions as a decompression chamber when the left atrial pressure and volume increase. ${ }^{6)}$ Patients who have disorders of sinus rhythm experience a decrease in the reservoir and conduit functions of the LA due to LAA closure or removal. ${ }^{7)}$ Furthermore, the reservoir-to-conduit ratio decreases, delaying the loading of the LA. ${ }^{7}$ LAA remodeling occurs in patients with AF. The changes that occur in the
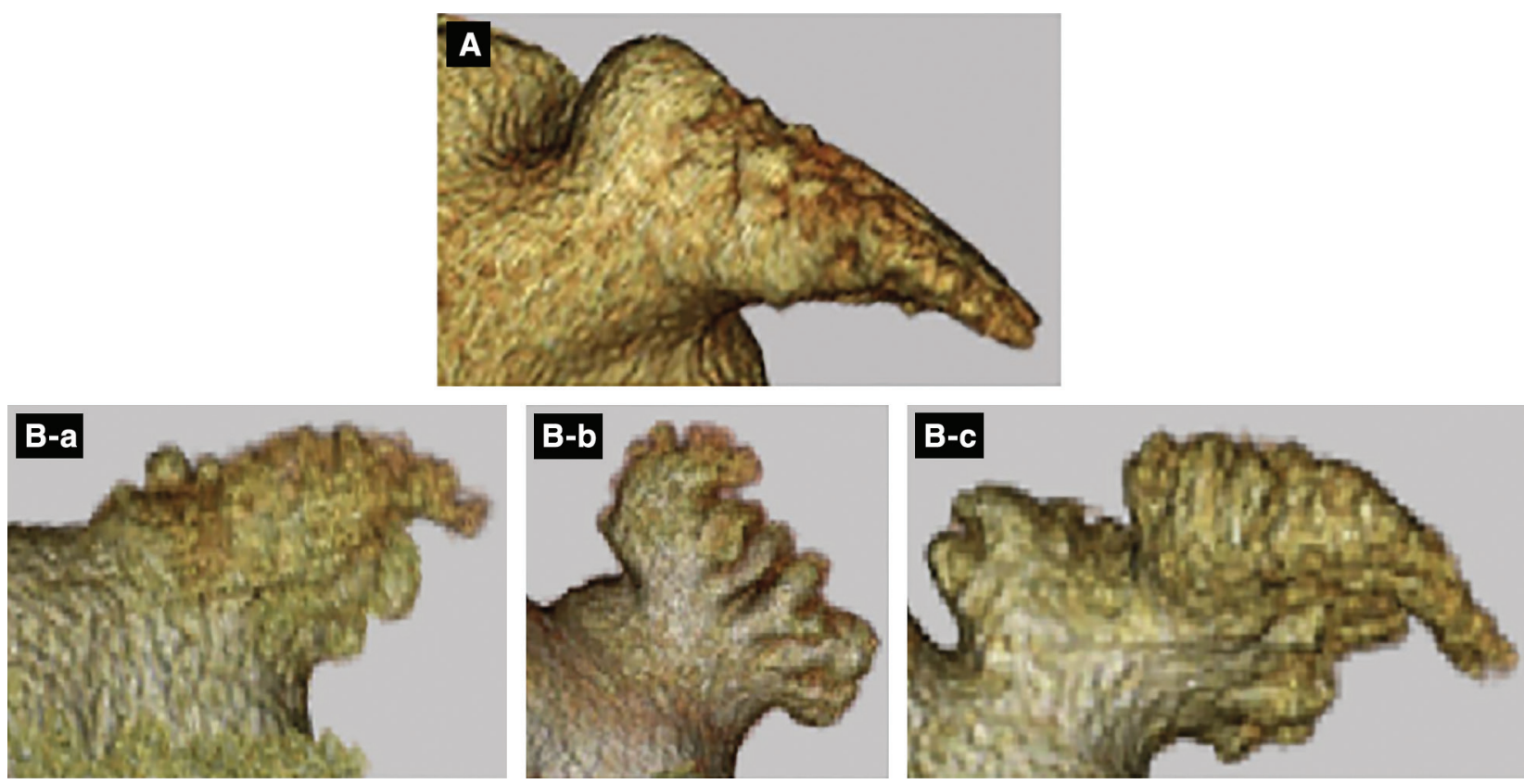

Fig. 3 3D-CT images of typical examples of LAA morphology. (A) Chicken wing type. (B) Different shapes of the non-chicken wing type: (B-a) cactus, (B-b) cauliflower, and (B-c) wind sock. ${ }^{3)}$ 3D-CT: three-dimensional computed tomography; LAA: left atrial appendage 
LAA, which functions as a static pouch, are accompanied by a decrease in the Doppler flow function and expandability, increasing the predisposition toward thrombus formation. However, these same changes occur in the sinus rhythm, following decreased cardiac and contractile functions of the LAA, and increased filling pressure. ${ }^{8)}$

\section{Functional Evaluation of the LAA}

Transesophageal echocardiography (TEE) is the most useful test for evaluating the LAA morphology and function. In the presence of the sinus rhythm, the LAA blood

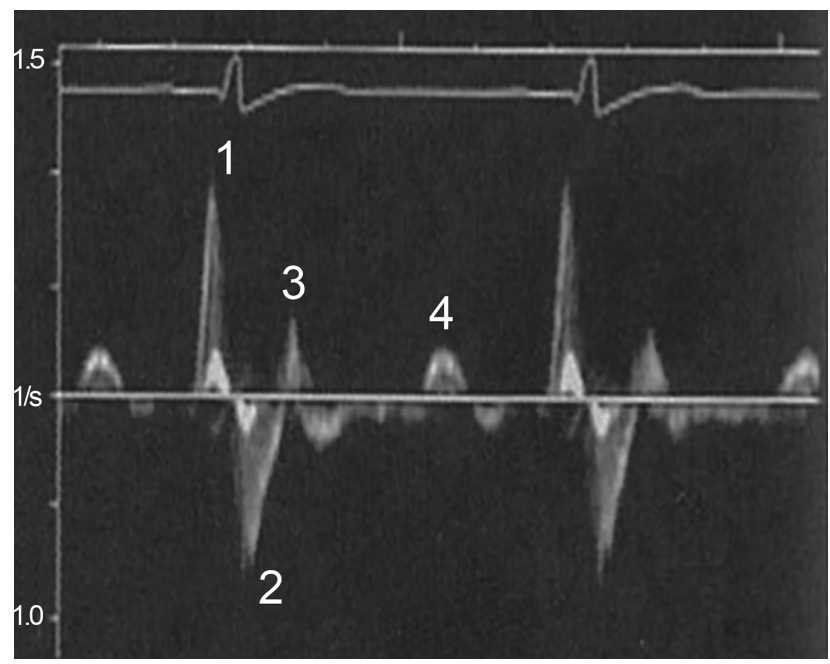

Fig. 4 Ultrasonic cardiogram findings. 1: LAA contraction. 2: Left atrial ears full. 3: Systolic reflected wave. 4: Early expansion of LAA outflow. ${ }^{6}$ LAA: left atrial appendage flow evaluated using the pulsed-wave Doppler has four phases (accelerated heart rate + two phases). Normally, a wave indicating inflow into the LA (occurring during ventricular diastole and coinciding with passive ventricular filling) and a wave indicating LAA filling are observed during LAA contraction. A systolic reflex wave (occurs when left atrial pressure increases during the ventricular systole) and a wave indicating outflow (from the LAA early in the diastole) are observed during bradycardia (Fig. 4). During AF, these LAA waveforms decrease and are classified according to different patterns (Fig. 5). ${ }^{9)}$

During sinus rhythm, the LAA contracts autonomously, but when AF occurs, its contractile ability declines, and blood can stagnate within the LAA, increasing the risk of thrombus formation. TEE has revealed that the decrease in contractile capacity during AF occurs due to the decreased blood flow velocity in the LAA. ${ }^{10,11)}$ Even when AF does occur, thrombi do not necessarily form subsequently. Nevertheless, it has been reported that thrombi may form within 3 days after the onset of AF. ${ }^{12)}$

\section{The Relation between the LAA and AF}

From the anatomical and electrical perspectives, the LAA is seldom associated with the etiology of AF. However, if AF is recurrent, anatomical (Fig. 6) and physiological remodeling ensue. ${ }^{13)}$ The resultant myocardial hypertrophy, ventricular fibrosis, and decreased expression of the apoptotic channel proteins of the cardiomyocytes lead to the involvement of the LAA in AF. ${ }^{14,15)}$ The
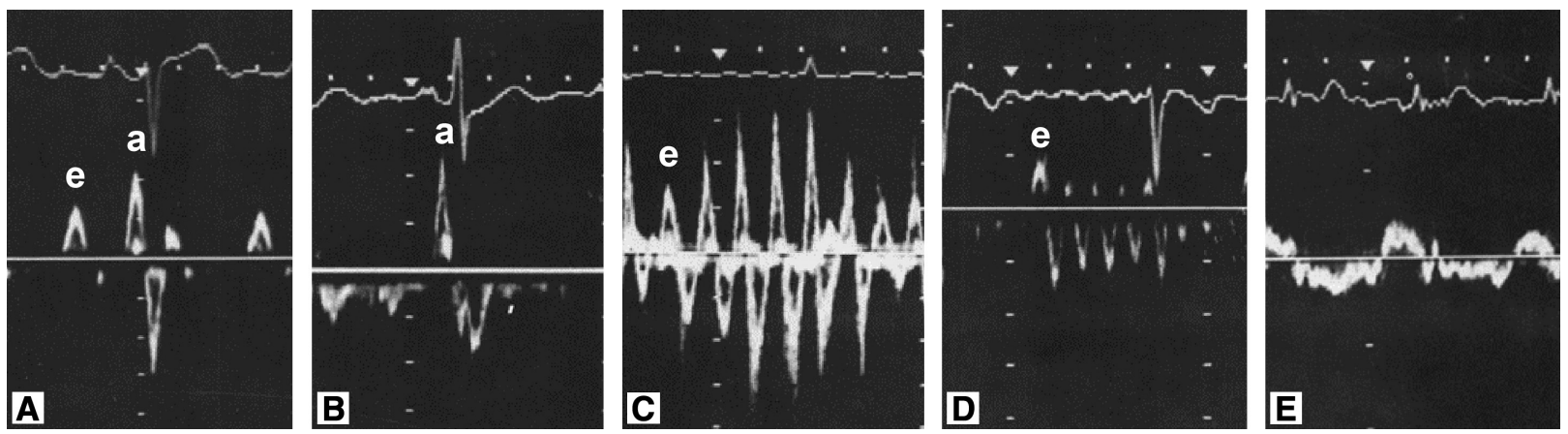

Fig. 5 Ultrasonic cardiogram findings. LAA blood flow velocity patterns. (A) Type I: a small e wave corresponded to mitral valve opening and an initial reduction in LAA diameter, followed by a larger wave immediately after the ECG P wave that corresponded to LAA contraction. (B) Type II: a single a wave, without a preceding e wave. (C) Type III: an outflow e wave followed by multiple irregular waves of higher velocity within each cardiac cycle associated with the vigorous fibrillatory motion of the LAA. (D) Type IV: the most frequently observed pattern comprising a dominant e wave with or without smaller fibrillatory waves and little fibrillatory motion of the appendage. (E) Type V: a prolonged low-velocity wave associated with minimal changes in the diameter of the LAA. ${ }^{9)}$ ECG: electrocardiographic; LAA: left atrial appendage 

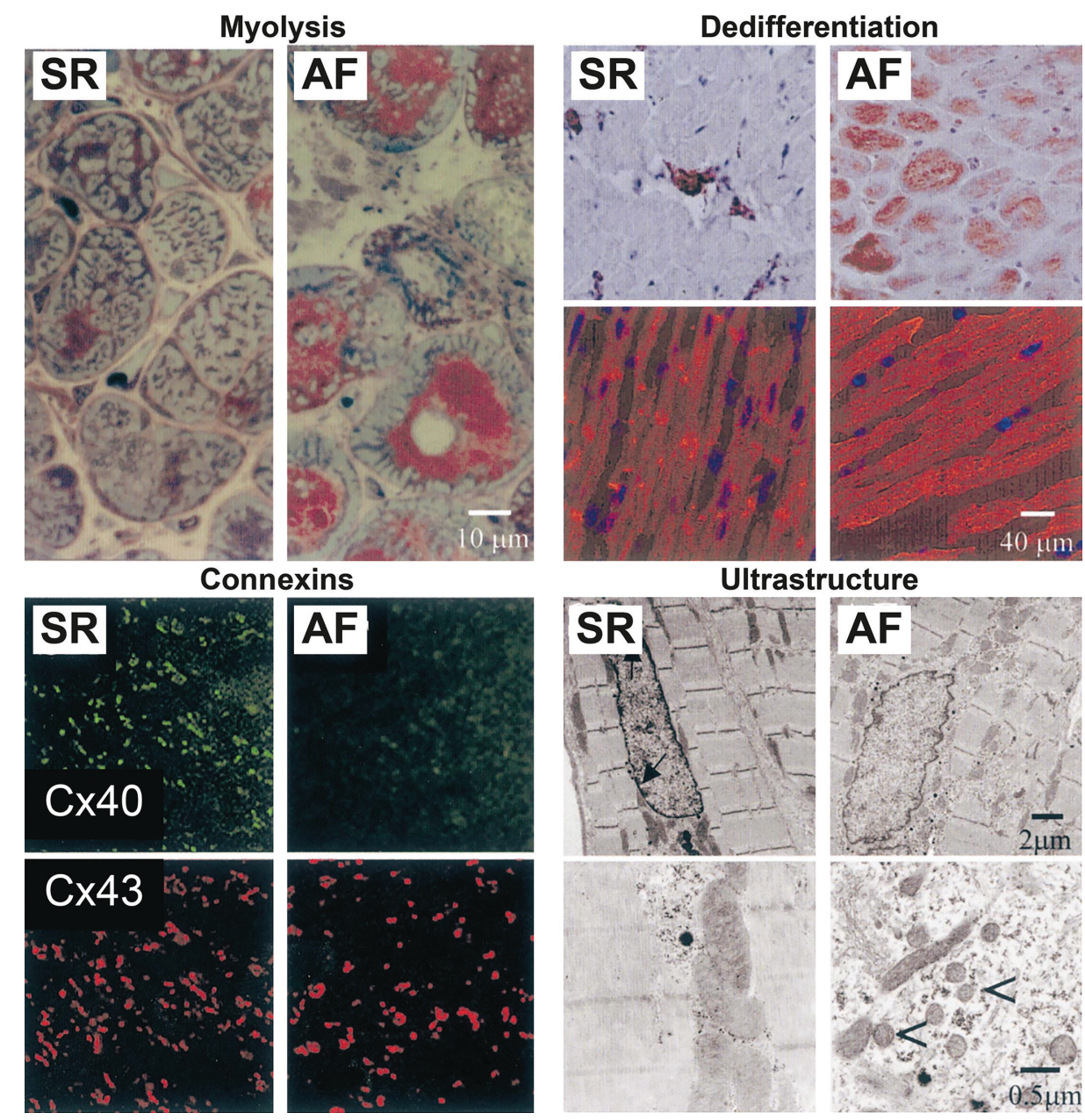

Fig. 6 Structural remodeling of the atrial myocytes after 4 months of AF in goats. Light microscopy (upper left panel) shows cells with severe myolysis (loss of sarcomeres: blue staining) and accumulation of glycogen (red). Immunostaining of structural proteins (right upper panel) demonstrates the dedifferentiation of the atrial myocardium by an apparent increase in fetal $\alpha$-smooth muscle actin (red staining in upper pictures). In the lower images of this panel, the myocytes were stained for desmin (red). Blue DAPI stains the nuclei. During AF, desmin loses its cross-striated pattern in the cytoplasm, and at the intercalated disks, the intensified desmin staining is no longer present. In the lower-left panel, changes in the gap junctions are shown. Labeling of Cx40 (green) and $\mathrm{Cx} 43$ (red) revealed an apparent reduction in $\mathrm{Cx} 40$ and no change in $\mathrm{Cx} 43$ expression. Electron microscopy (lower right) shows changes in the subcellular organization of atrial myocytes. During AF, the atrial nuclei have a more homogeneous distribution of chromatin. For comparison, the standard clustering of chromatin at the nuclear membrane is indicated by arrows in the upper left panel. During AF, many small donut-shaped mitochondria can be found (arrowheads right lower panel). ${ }^{13)}$ AF: atrial fibrillation; DAPI: 4',6-diamidino-2-phenylindole

ganglionated plexi (GP), located in the vicinity of the LAA, the PV, and the adipose tissue also contribute to this remodeling.

The heart's autonomic innervation is divided into extrinsic and intrinsic. The extrinsic innervation refers to the vagus nerve and sympathetic nerves distributed from the brain and spinal cord to the heart. Intrinsic innervation refers to the GP located in the pericardial region. The GP contains a fixed ratio of parasympathetic and sympathetic nerves, with both having an interactive effect on the atrial muscles (Figs. 7 and 8). Since the GP acts independently on the atrial muscles, they are known as the "little brains of the heart." They are distributed mainly in five locations in the vicinity of the LA; the superior left GP located at the canopy of the inlet of the superior left PV, the Marshall tract GP situated between the left PV inlet anterior wall and the LAA, the anterior right GP located in the anterior wall of the inlet of the right PV, the inferior left GP located from the inferior margin of the inferior left PV to the left side of the LA base, and the inferior right GP located from the inferior margin of the inferior right $\mathrm{PV}$ to the right side of the LA base. Currently, accurate mapping of the exact 


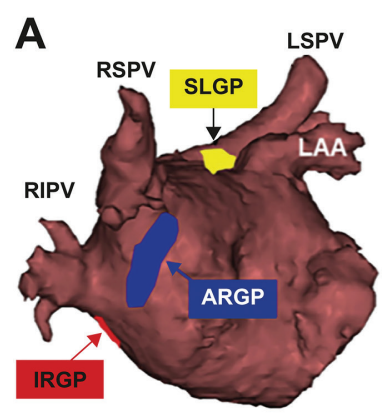

RAO View

B

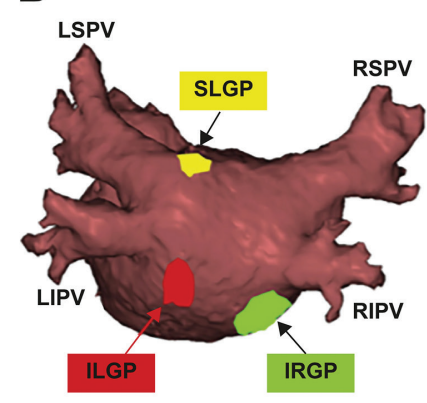

PA View

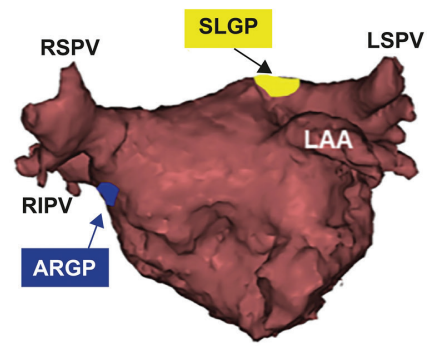

AP Cranial View

Fig. 7 Usual locations of the four major atrial GP. (A) The SLGP is located near the junction of the LSPV and LA. The anterior right GP is located near the anterior aspect of the RSPV-LA junction. ARGP is not located on the posterior wall of the LA. (B) The IRGP is situated at the LA's inferior aspect, below the lower edge of the RIPV. The ILGP is situated at the inferior aspect of the posterior wall of the LA. The location of the ILGP can be adjacent to the LIPV (left) or $>2 \mathrm{~cm}$ away from the inferior edge of the LIPV (right). ARGP: anterior right GP; GP: ganglionated plexi; ILGP: inferior left GP; IRGP: inferior right GP; LA: left atrium; LAA: left atrial appendage; LIPV: left inferior pulmonary vein; LSPV: left superior pulmonary vein; PA: pulmonary artery; RAO: right anterior oblique; RIPV: right inferior pulmonary vein; RSPV: right superior pulmonary vein; SLGP: superior left GP. ${ }^{16)}$

locations of each of these is in progress, enabling more precise ablation. ${ }^{16,17)}$

The concept of pathophysiological changes in the atria, LAA, PV, and pericardial tissue has been conceptualized as "atrial myopathy" based on the remodeling and subsequent changes taking place as each of these elements has its effect (Fig. 9A and 9B). ${ }^{18)}$

\section{Rate of Transition to AF}

A previous study reported that $3.3 \%$ of the population of the United States (US) suffered from AF with

an expected 1.5-fold increase in figures over the next 20 years. ${ }^{19)}$ The Framingham heart study reported a $25 \%$ risk of $\mathrm{AF}$ among those aged 40 years and older. ${ }^{20)}$ $\mathrm{AF}$ is the most common type of heart arrhythmia, with 32 million sufferers worldwide and 5 million sufferers in the US, which incurs an estimated annual medical cost of 26 billion US dollars. ${ }^{21)}$ There have been many reports from Asia as well, and a survey of 96,841 people in Japan over 7 years revealed that the incidence rate of AF was 5.3\%.22) This study scored the following independent risk factors: age, sex (male), waist circumference, diastolic blood pressure (BP), amount of alcohol intake, heart rate, and change in electrocardiogram variables, to identify individuals at high risk of incident AF. An observational study of 471,466 people over 11 years (mean: 4.1 years) in China, excluding individuals with valvular disease, reported the occurrence of $\mathrm{AF}$ at a rate of $0.5 / 1000$ person-years. ${ }^{23)}$ In this study, cases of valvular disease were excluded as many of these cases transitioned to AF. A score was developed based on independent risk factors including coronary heart disease, chronic obstructive pulmonary disease, hypertension, heart failure, age ( $\geq 75$ years), and thyroid disorders. It was found that with an increase in score, the onset rate of AF also increased, and the score had a better predictivity than the congestive heart failure, hypertension, age $>75$ years, diabetes (all 1 point each); previous stroke (2 points) $\left(\mathrm{CHADS}_{2}\right)$ scores.

At this medical facility, follow-up investigations of 2822 patients who underwent coronary artery bypass grafting (CABG) (mean follow-up period: 5.8 years) indicated that the transition rate to AF over 10 years was $10.2 \%$ in postoperative sinus rhythm populations and $23.1 \%$ in postoperative atrial fibrillation populations (POAF) (Fig. 10A). Early POAF after CABG is an independent risk factor for late-onset $\mathrm{AF}$ (hazard ratio 2.80, $95 \%$ confidence interval $1.86-4.22, \mathrm{p}<.001$ ), but further follow-up examination and investigation of predicted risk factors are required (Fig. 10B). ${ }^{24)}$ In terms of preoperative risk, the rate of conversion to $\mathrm{AF}$ at 10 years after surgery was $5 \%$ or less for patients with a $\mathrm{CHADS}_{2}$ score of up to $3,8 \%$ for those with a score of 4 , and $41 \%$ for those with a score of 5 or higher. Since almost all patients received beta-blockers (bisoprolol fumarate) and statins after surgery, the transition to AF was considered to be suppressed in the low-score group. Further follow-up, prediction, and investigation of each risk factor are needed in this matter. 

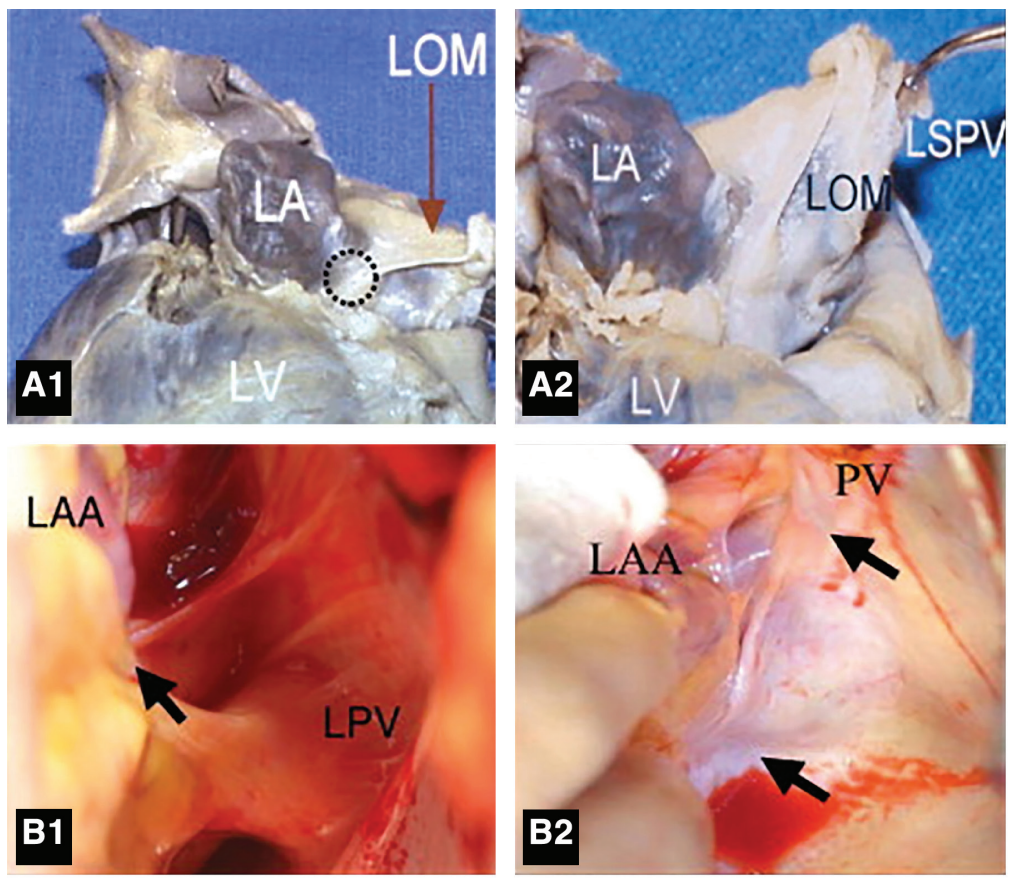

Proximal Connection

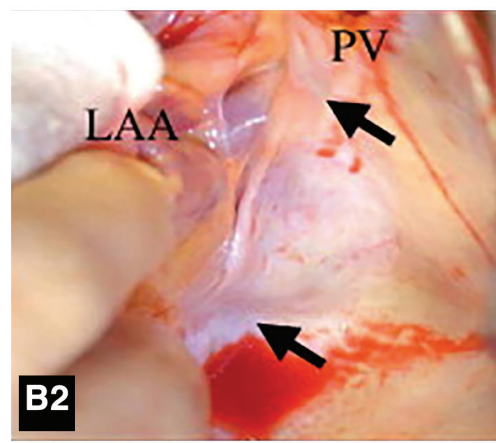

Double Connections
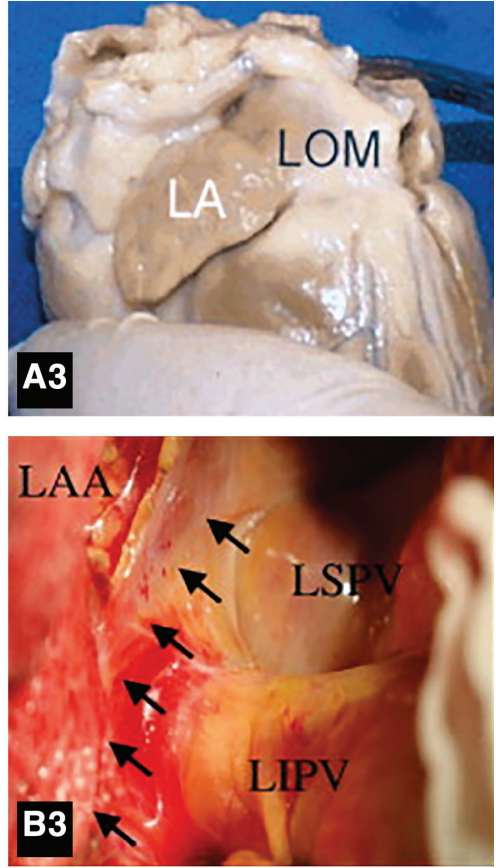

Multiple Connections

Fig. 8 Variations in the LOM anatomy. A1: The black circle indicates the proximal connection of the LOM to the CS. A2: A different view of the same heart. The distal end of the LOM inserts into the LSPV. A3: A second heart in which the LOM was completely attached to the epicardium. A discrete ligament was not identified. B1: Proximal connection (arrow) between LOM and CS. B2: Both the proximal and distal connections of the LOM (2 arrows) in the second heart. B3: A third heart, which seems to have multiple muscle fibers (arrows) connecting the LOM and LA. ${ }^{17)}$ LA: left atrium; LOM: ligament of Marshall; LSPV: left superior pulmonary vein

\section{AF and Cerebral Infarction}

When AF occurs, cardiac function decreases. This, in turn, increases the risk of thromboembolism due to intraatrial blood retention and left atrial thrombus. The occurrence of cerebral infarction in such cases is high, that is, between $6 \%$ and $24 \% .{ }^{25}$ ) The percentage of patients hospitalized for acute ischemic stroke (AIS) who suffered from AF increased from 20\% in 2004 to 24\% in 2013. Although the mortality rate for AIS with AF has decreased from $11.6 \%$ to $8.3 \%$ compared to the non-AF group, the medical costs incurred by patients with $\mathrm{AF}$ during hospitalization increased by 2310 US dollars and the length of hospital stay increased by 1.1 days. ${ }^{26)}$

A remote survey of 1733 non-rheumatoid AF patients between 65 and 95 years of age who did not undergo anticoagulant therapy revealed that 94 patients were re-admitted to the hospital due to the AIS (stroke rate: 4.4/100 patient-years). For each point by which the $\mathrm{CHADS}_{2}$ score increased, the cerebral infarction rate increased 1.5-fold and the CHADS 2 score of 1 increased the rate to $2.8 / 100$ patient-years. Therefore, when the
$\mathrm{CHADS}_{2}$ score exceeds 1, anticoagulant therapy should be considered and when it exceeds 2 , anticoagulant therapy is advisable. ${ }^{27)}$

A study of postoperative AIS following open-heart surgery reported that $1.8 \%$ of 108711 patients who underwent open-heart surgery experienced a perioperative stroke and $3.6 \%$ experienced stroke within 2 years following surgery. The most powerful predictive factors for early- and delayed-onset stroke were age $(\geq 65$ years), history of stroke or transient ischemic attack (TIA), peripheral vascular disease, combined coronary artery bypass surgery, and valvular disease surgery. The preoperative dialysis and newly occurring postoperative $\mathrm{AF}$ were predictive factors only for early-onset stroke, and a $\mathrm{CHADS}_{2}$ score of 2 or higher was related to increased risk of stroke or death (Fig. 11A and 11B). ${ }^{28)}$

\section{AF and Anticoagulant Therapy}

Currently, oral anticoagulant (OAC) therapy or direct oral anticoagulant (DOAC) therapy with warfarin is 
$\mathbf{A}$

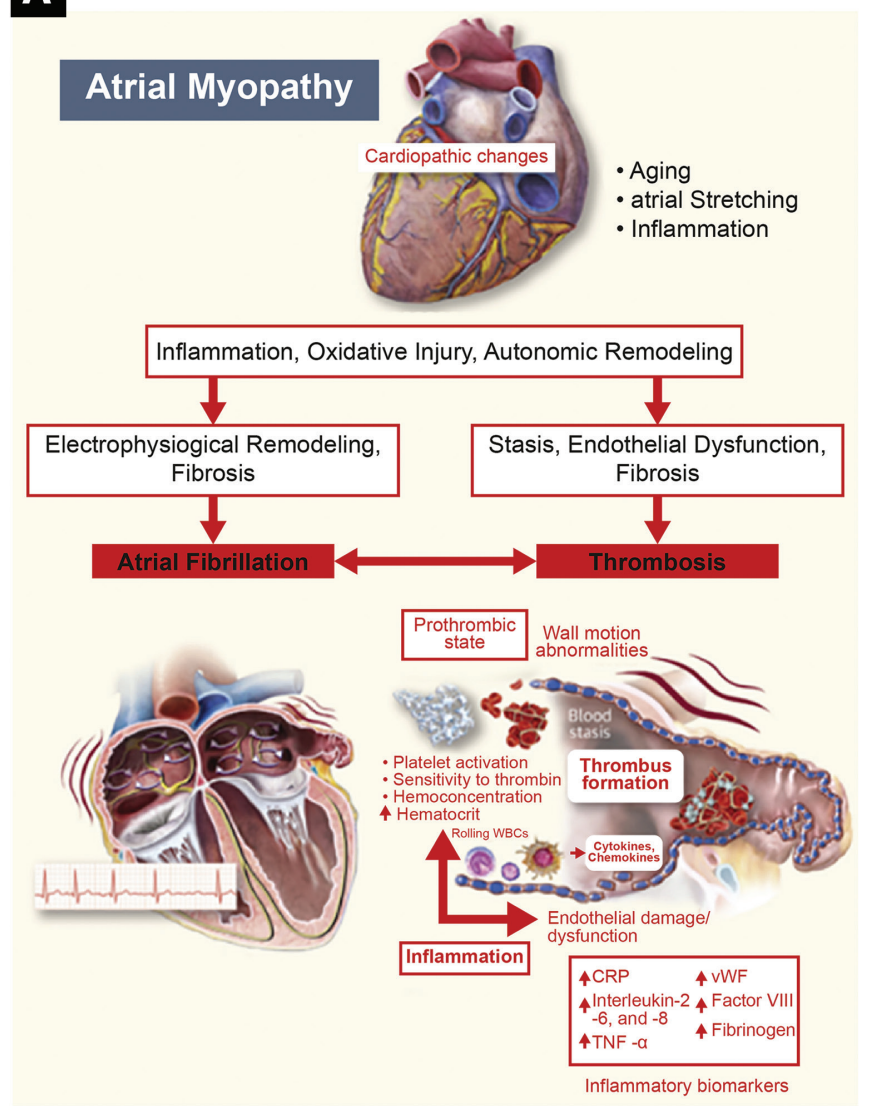

B

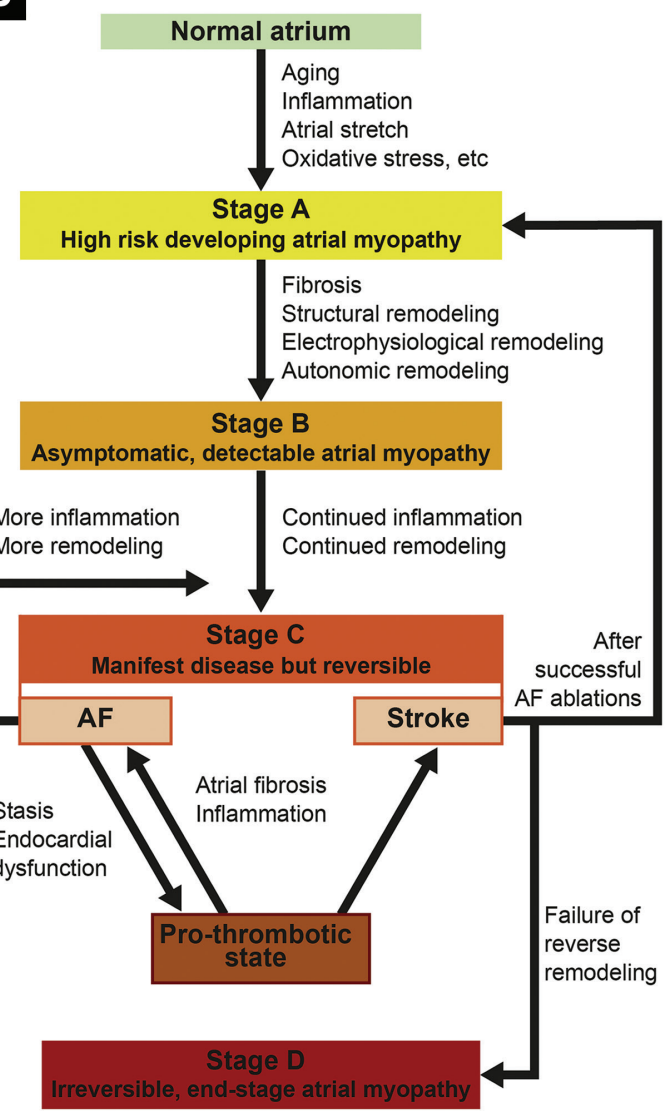

Fig. 9 Atrial myopathy. (A) Atrial myopathy and the relationship between AF and stroke. Atrial myopathy is typically caused by insults such as aging, inflammation, oxidative stress, and atrial stretch. Furthermore, atrial myopathy results in endothelial dysfunction and stasis, thereby producing a prothrombotic state. Electrophysiological remodeling and fibrosis facilitate the development of $\mathrm{AF}$, which leads to inflammation, fibrosis, and autonomic remodeling, all of which contribute to a worsening prothrombotic environment, mediated by circulating inflammatory cytokines, chemokines, and other molecules such as CRP, IL-2, -6 , and -8, TNFa, etc. AF and thrombosis can develop separately and interact closely to further aggravate the underlying atrial myopathic processes. (B) Stages of atrial myopathy. ${ }^{18)}$ AF: atrial fibrillation; CRP: C-reactive protein; IL: interleukin; TNF: tumor necrosis factor; vWF: von Willebrand's factor; WBC: white blood cell

thought to be optimal for the prevention of stroke and systemic thromboembolism in patients with $\mathrm{AF}$ or a $\mathrm{CHADS}_{2}$-VASc score (congestive heart failure, hypertension, age $\geq 75$ years). OAC consistently improves the survival rate in those with diabetes, stroke, TIA, peripheral vascular disease, age of 65-74 years, and in women. ${ }^{29,30)}$ However, OAC is either absolutely or relatively contraindicated for most patients who are at risk of stroke due to the high risk of bleeding, discontinuation of oral medications, and use of other medications. Approximately $45 \%$ of all patients who undergo OAC therapy (including DOAC) discontinue treatment within 2 years. $^{31,32)}$

Although anticoagulant therapy is considered essential for preventing stroke, it is often challenging to remain compliant in everyday life. Many patients are unable to maintain this therapy; thus, a new concept for stroke prevention is required.

\section{LAA Management for Stroke Prevention}

Surgical amputation of the LAA is one of the oldest surgical procedures, first performed in 1949. Subsequently, many procedures have been developed, producing favorable results as well as causing several problems. As previously mentioned, the "non-chicken wing" types, especially the cauliflower type of LAA, are most often related to cardiogenic embolism. Therefore, it is critical to devise a method to deal with this morphological type of LAA. 
Yamamoto T, et al.

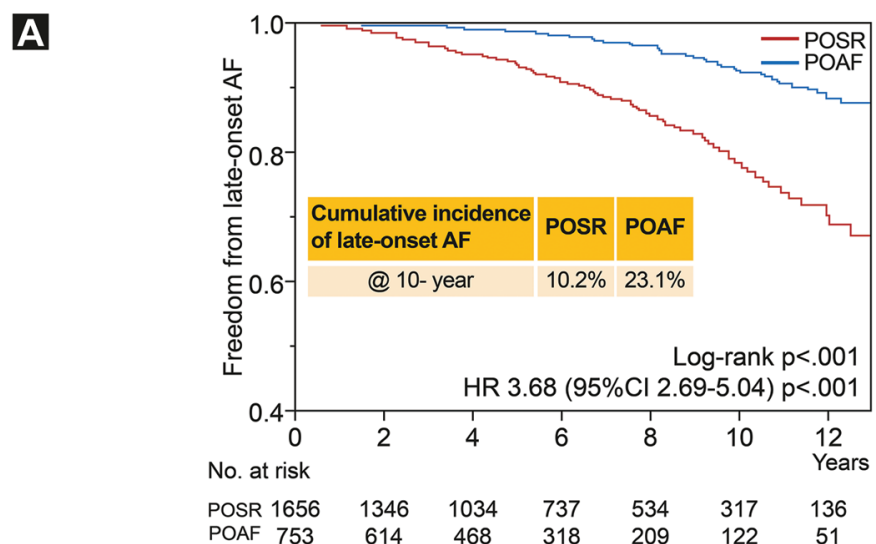

B

\begin{tabular}{|l|cccc|}
\hline \multirow{2}{*}{ Cox Proportional Hazard Model } & \multicolumn{3}{c|}{ Univariate } & \multicolumn{2}{c|}{ Multivariate } \\
\cline { 2 - 5 } & $\mathrm{HR}(95 \% \mathrm{Cl})$ & $\mathrm{P}$ value & $\mathrm{HR}(95 \% \mathrm{Cl})$ & $\mathrm{P}$ value \\
\hline Age & $1.03(1.00-1.05)$ & 0.03 & $1.02(0.99-1.05)$ & 0.07 \\
Male sex & $0.88(0.55-1.40)$ & 0.58 & & \\
BMIl>30 & $0.85(0.12-6.22)$ & 0.84 & & \\
Hypertension & $1.23(0.50-3.01)$ & 0.65 & & \\
Dyslipidemia & $0.77(0.52-1.14)$ & 0.20 & & \\
Diabetes & $1.05(0.73-1.51)$ & 0.73 & & \\
Chronic kidney disease (eGFR<60) & $1.65(1.15-2.38)$ & $<0.01$ & $1.44(0.99-2.09)$ & 0.06 \\
Hemodialysis & $2.74(1.54-4.87)$ & $<0.01$ & & \\
Previous stroke & $2.33(1.55-3.50)$ & $<0.01$ & $2.05(1.35-3.10)$ & $<0.01$ \\
Peripheral arterial disease & $1.32(0.85-2.04)$ & 0.22 & & \\
Ejection fraction < 50\% & $1.19(0.79-1.79)$ & 0.40 & & \\
Left atrial appendage amputation & $1.22(0.70-2.13)$ & 0.47 & & \\
POAF & $2.82(1.88-4.24)$ & $<0.01$ & $2.80(1.86-4.22)$ & $<0.01$ \\
Isolated CABG & reference & & reference & \\
\hline Concomitant Aortic valve surgery & $1.44(0.84-2.47)$ & 0.18 & $1.30(0.75-2.23)$ & 0.35 \\
Concomitant Mitral valve surgery & $1.40(0.65-.3 .03)$ & 0.39 & $1.37(0.63-2.99)$ & 0.43 \\
\hline Concomitant Aortic surgery & $3.12(1.53-6.61)$ & $<0.02$ & $2.48(1.19-5.16)$ & 0.01 \\
\hline
\end{tabular}

Fig. 10 (A) Event-free survival curve of late-onset AF after CABG. (B) Risk factors associated with late-onset AF. ${ }^{24)}$ AF: atrial fibrillation; CABG: coronary artery bypass grafting; POAF: postoperative atrial fibrillation; POSR: postoperative sinus rhythm

The 2017 Society of Thoracic Surgeons guidelines recommend LAA closure as class IIa. ${ }^{33)}$ In particular, they determined that LAA procedures are optimal and are required mostly in patients who are at high risk of stroke and for whom anticoagulants are contraindicated.

A study reported the results during a mean follow-up period of 2.1 years after LAAO which was performed on 4374 of $75782(5.8 \%)$ patients who underwent heart surgery. It was found that LAAO clearly contributed to a decrease in stroke and that simultaneously performed as open-heart surgery led to a decrease in both stroke and overall mortality rate (AF patients: $75 \%$; non-AF patients; 25\%). However, in this study, the effectiveness of LAAO on patients with no preoperative history of AF was not verified (Fig. 12). ${ }^{34}$

\section{Percutaneous LAA Closure Device}

The use of LAA closure with a catheter for patients with AF alone and without other heart-related complications is becoming widespread as it is markedly less invasive than surgery.

Many studies have reported clinical outcomes obtained using the Watchman (WM) device (Boston Scientific, Atritech, Plymouth, MN, USA), which has been in use for an extended period. A meta-analysis reported that, although the patients who were administered warfarin for ischemic stroke showed favorable results, there were significantly fewer cases of hemorrhagic stroke in the WM group. The observation of all strokes indicated no significant difference between the two groups, and the WM was reported to be noninferior to warfarin (Fig. 13) ${ }^{35)}$ A recent study on the cost-effectiveness of warfarin, the major form of conventional anticoagulant therapy, and two other therapies (DOAC, percutaneous LAA closure [P-LAAC]) reported that the per-patient cost in the P-LAAC group was lower than that in the DOAC and warfarin groups in the fifth and tenth year postoperatively, respectively (Fig. 14). ${ }^{36)}$ 
$\mathbf{A}$

\begin{tabular}{|c|c|c|}
\hline \multirow{2}{*}{ Variable } & \multicolumn{2}{|c|}{ Adjusted $\mathrm{HR}^{*}(95 \% \mathrm{Cl})$} \\
\hline & Men & Women \\
\hline$A g e \geq 65 \mathrm{v} .<65 \mathrm{yr}$ & $2.1(2.0-2.2)$ & $1.6(1.5-1.7)$ \\
\hline Previous stroke or transients ischemic attack & $1.6(1.5-1.8)$ & $1.4(1.2-1.6)$ \\
\hline Dialysis & $2.8(2.5-3.2)$ & $2.7(2.2-3.3)$ \\
\hline Peripheral vascular disease & $1.6(1.5-1.7)$ & $1.5(1.4-1.7)$ \\
\hline Creatinine $\geq 90 \mathrm{v} .<90 \mu \mathrm{mol} / \mathrm{L}$ & $1.1(1.0-1.1)$ & $1.1(1.1-1.2)$ \\
\hline History of myocardial infarction & $1.0(1.0-1.1)$ & $1.1(1.0-1.2)$ \\
\hline Congestive heart failure & $1.9(1.8-2.0)$ & $1.8(1.7-1.9)$ \\
\hline History of atrial fibrillation & $1.4(1.3-1.5)$ & $1.3(1.2-1.4)$ \\
\hline Left main coronary artery disease & $1.3(1.2-1.3)$ & $1.4(1.2-1.5)$ \\
\hline LV dysfunction grade $2-4$ v. grade 1 & $1.3(1.2-1.4)$ & $1.2(1.2-1.3)$ \\
\hline Reoperation & $1.4(1.3-1.6)$ & $1.4(1.2-1.6)$ \\
\hline Postoperative atrial fibrillation & $1.1(1.0-1.1)$ & $1.0(0.9-1.1)$ \\
\hline CABG and valve surgery v. CABG alone & $1.7(1.6-1.9)$ & $1.9(1.7-2.1)$ \\
\hline Valve surgery v. CABG & $1.4(1.3-1.5)$ & $1.3(1.2-1.5)$ \\
\hline $\begin{array}{l}\text { Note: } \mathrm{CABG}=\text { coronary artery bypass grafting, } \mathrm{Cl}=\mathrm{c} \\
\mathrm{LV}=\text { left ventricular. } \\
{ }^{*} \text { Adjusted for all other variables in the table }\end{array}$ & & \\
\hline
\end{tabular}

B

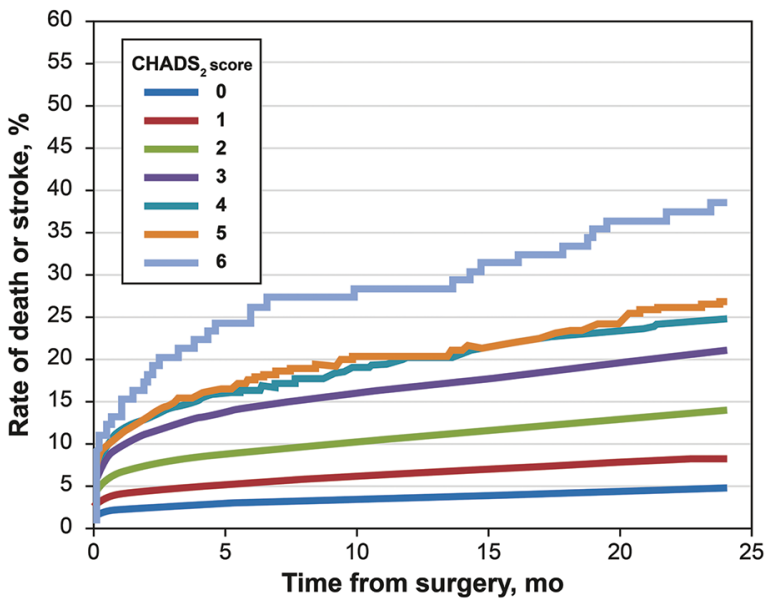

Fig. 11 (A) Predictors of death or stroke within 2 years after cardiac surgery, based on sex. (B) Rate of stroke or death from the time of hospital admission for cardiac surgery to 2 years after surgery. Rate of stroke or death from the time of hospital admission for cardiac surgery to 2 years after surgery, based on the $\mathrm{CHADS}_{2}$ score. $\mathrm{CHADS}_{2}$ : congestive heart failure, hypertension, age $>75$ years, diabetes mellitus, and prior stroke or transient ischemic attack; 1 point is given for the presence of each of the first 4 conditions and 2 points for a history of stroke or transient ischemic attack. ${ }^{28)}$

In Japan, a clinical study was conducted to assess the safety and effectiveness of P-LAAC in Japanese patients. It reported on the safety of the SALUTE trial and that the LAA closure was safe and effective. This was comparable to the results of other large-scale non-randomized clinical studies. Thus, LAA closure using a catheter has been demonstrated to be effective in Japanese patients requiring anticoagulant therapy for $\mathrm{AF}^{37}{ }^{37}$

\section{Special Cases}

\section{Dialysis patients}

Theoretically, LAAO is considered an effective procedure for high-risk patients. Currently, most LAA studies exclude dialysis patients, but an observational study of patients with chronic kidney disease (including those on dialysis) who underwent LAAO using a transcatheter self-expanding nitinol plug reported favorable results with limited complication rates. ${ }^{38)}$ Although further studies are required to determine the safety and effectiveness of this LAA closure device used in end-stage kidney disease patients, it is believed to be an effective treatment method.

\section{Patients with high $\mathrm{CHADS}_{2}$-VASc scores}

The ROCKET AF study of patients with high $\mathrm{CHADS}_{2}$-VASc scores reported the occurrence of strokes at a rate of 5/100 patient-years in the group with $\mathrm{CHADS}_{2}$-VASc scores of $\geq 5$ over 2 years. ${ }^{39)}$ There were no hemorrhagic contraindications for the use of OAC 
Yamamoto T, et al.
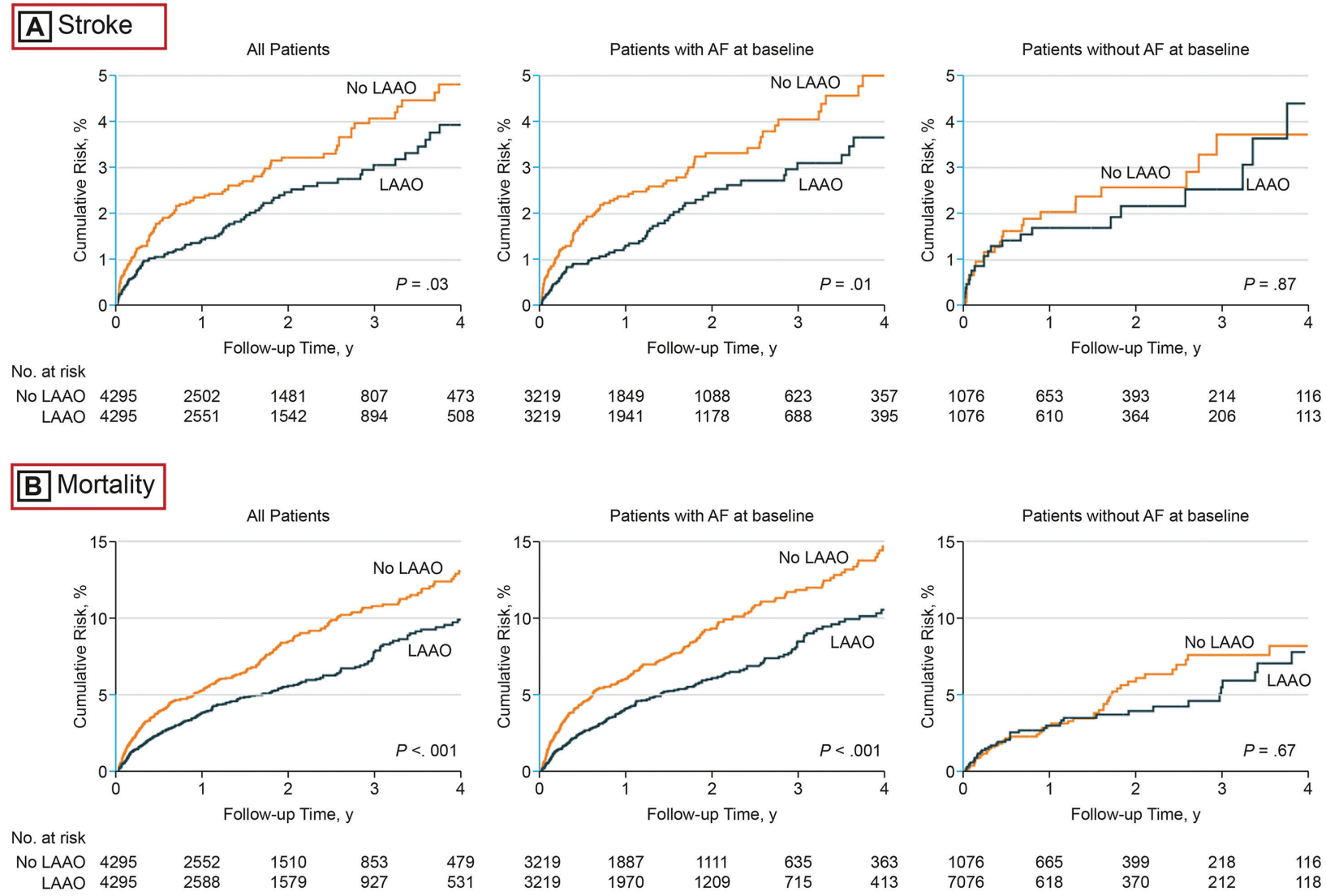

Fig. 12 Association of surgical LAAO with subsequent stroke and mortality among patients undergoing cardiac surgery. ${ }^{34)}$ LAAO: left atrial appendage occlusion

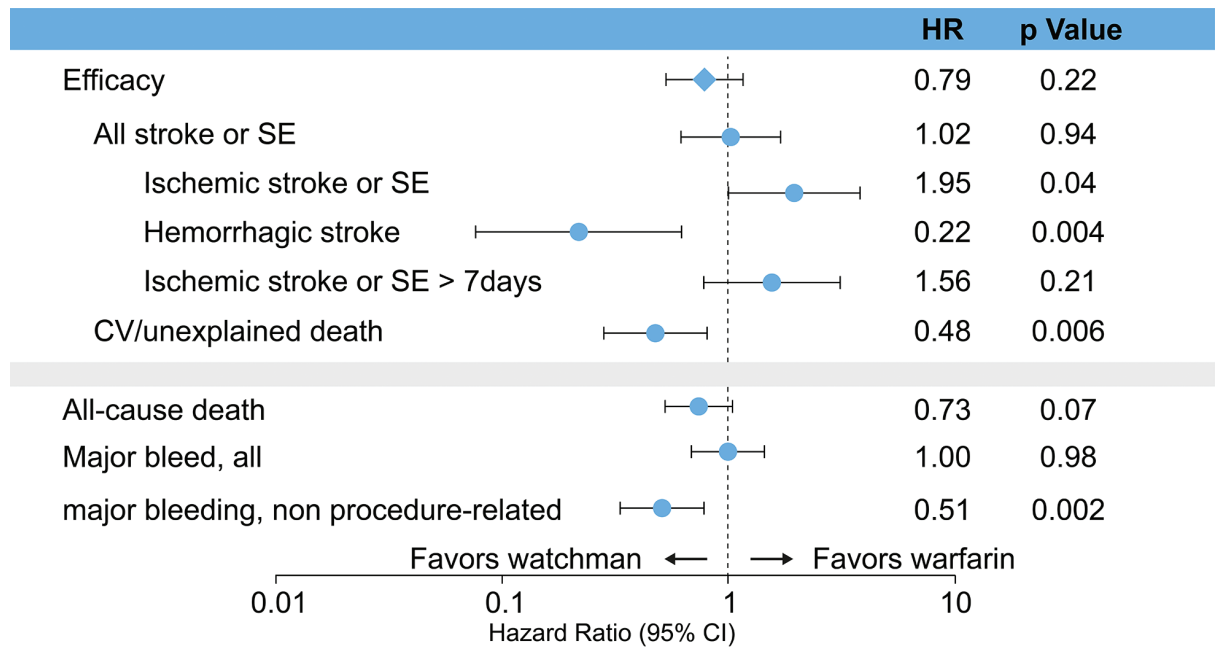

Fig. 13 LAA closure as an alternative to warfarin for stroke prevention in $\mathrm{AF}^{35)} \mathrm{LAA}$ : left atrial appendage

therapy in these patients, but combined therapy consisting of LAA closure and OAC might be beneficial. Currently, no data have indicated the usefulness of combined therapy, but it is advisable for patients in high-risk groups.

\section{Problems Associated with LAA Closure}

LAA closure is associated with several challenges. These issues can be classified as postoperative anticoagulant therapy, device-related thrombi, postprocedural 


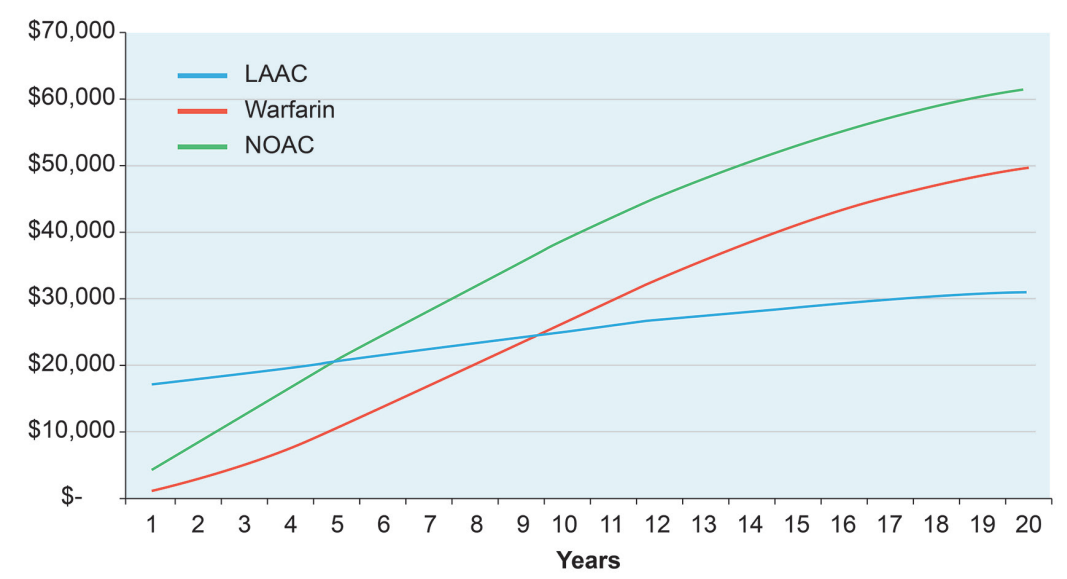

\begin{tabular}{|c|c|c|c|}
\hline & $\begin{array}{l}\text { Time to Clinical Effectiveness } \\
\text { (Incremental QALYs) }\end{array}$ & $\begin{array}{l}\text { Time to Cost-Effectiveness } \\
\text { (Cost per QALY) }\end{array}$ & $\begin{array}{l}\text { Time to Dominance } \\
\text { (More Effective, Less Costly) }\end{array}$ \\
\hline LAAC vs. warfarin & $\begin{array}{l}\text { Years } 3 \\
(0.015)\end{array}$ & $\begin{array}{c}\text { Years } 3 \\
\text { (\$42,994/QALY) }\end{array}$ & Years 3 \\
\hline NOACs vs. warfarin & $\begin{array}{l}\text { Year } 1 \\
(0.008)\end{array}$ & $\begin{array}{c}\text { Year } 1 \\
\text { (\$48,446/QALY) }\end{array}$ & N/A \\
\hline LAAC vs. NOACs & $\begin{array}{l}\text { Year } 5 \\
(0.007)\end{array}$ & $\begin{array}{c}\text { Year } 5 \\
\text { (Dominant) }\end{array}$ & Year 5 \\
\hline
\end{tabular}

Fig. 14 Time to cost-effectiveness following stroke reduction strategies in AF (warfarin, NOAC, and LAAC). Upper panel: Average total cumulative costs per patient over 20 years. LAAC becomes less costly than NOACs and warfarin at 5 and 10 years, respectively. Lower panel: Summary of time to clinical and cost-effectiveness for each treatment arm. LAAC becomes cost-effective at 7 and 5 years relative to warfarin and NOACs, respectively. NOACs achieve cost-effectiveness relative to warfarin at 16 years. ${ }^{36}$ LAAC: left atrial appendage closure; NOAC: non-warfarin oral anticoagulants

leaks, and problems associated with embolization procedures using devices.

\section{Postoperative anticoagulant therapy}

The biggest challenge associated with P-LAAC is assessing the bleeding risk associated with postoperative anticoagulant therapy. Recent studies have reported the rate of device-related thrombi using endocardium devices within a range of $3-7.2 \%{ }^{40,41)}$ The use of an LAA extracardiac closure system and direct surgical amputation share the advantage of not introducing foreign objects into the blood vessels, which would considerably lower the risk of thrombi formation. Nevertheless, with the improvement of our understanding of the mechanism of coagulation and bleeding at the cellular and molecular levels, endocardium closure procedures utilizing next-generation catheters may dramatically transform clinical treatment.

\section{Postprocedural leaks}

Although remnant intra-LAA blood flow is often observed after P-LAAC, it is rarely observed after the use of the LAA extracardiac closure system or after surgical amputation of the LA. It has been reported that the rate at which remnant blood flow is observed is between 0 and $63 \%$, depending upon the type of LAA device utilized, the frequency of monitoring, and the modality. ${ }^{42,43)}$

In a previous study, of 2546 patients who underwent surgical LAA closure, 137 patients (5.3\%) underwent postoperative course observation using TEE. The TEE assessment period was $8.1 \pm 12$ months post-surgery. The success rate of the LAA was $40 \%$ (55/137 patients), and although remnant blood flow and stump/pouch that exceeded $1 \mathrm{~cm}$ were observed in cases of LAA closure using a stapler, in cases of the closure via endocardium suturing, 25\% had remnant blood flow. ${ }^{44)}$ In addition, a study in which the postoperative course following LAA closure and ligation was performed in 72 patients with a $\mathrm{CHADS}_{2}$-VASc score of $4.1 \pm 1.9$ reported that overall $64 \%$ of patients experienced complete closure, $24 \%$ experienced remnant blood flow, and $12 \%$ experienced partial remnant LAA stump. The long-term stroke and embolism rates were $2 \%, 24 \%$, and $0 \%$ in cases with complete closure, remnant blood flow, and remnant stump, respectively. None of the patients who 
experienced postoperative embolism had undergone anticoagulant therapy and all had recurrent AF during the follow-up period. ${ }^{45}$ Remnant blood flow into the LAA is critical once the engorged neck region reaches below $5 \mathrm{~mm}$; hence, the continuation of anticoagulant therapy is advisable.

Based on a variety of data that have been reported until now, the importance of determining the appropriate size of the device and that of diagnostic imaging has become clear. The issues of confirming the presence of postoperative remnant blood flow and preventing delayed-onset stroke are of utmost importance.

\section{Device-related thrombi}

Device-related thrombi are rare. There are challenges associated with nitinol cage P-LAAC devices, transcatheter self-expanding nitinol plugs, and other types of endocardium LAA devices, and the rate of occurrence is reported to be $4 \% .{ }^{46)}$ Many device-related thrombi occurred during the postoperative hospital stay (65\%), but $\sim 30 \%$ were associated with late embolization. In patients who experienced device-related problems, most devices could be removed percutaneously, ${ }^{47)}$ but some complex cases required a surgical approach. Operators with little experience should understand the anatomical structure of the LAA through both TEE and 3D-CT to select the appropriate device to minimize complications.

\section{Endocrine Changes Caused by LAA Amputation}

Recently, the effects of endocardial and epicardial LAA procedures on natriuretic peptides have been analyzed and reported. Lakkireddy et al. ${ }^{48)}$ compared serum aldosterone, adrenaline, noradrenaline, ANP, and brain natriuretic peptide (BNP) at baseline, immediately after the procedure, and at 3 months postoperatively in patients who underwent LAA exclusion using epicardial and endocardial procedures. The results indicated that epicardial LAA devices led to a significant downregulation of adrenaline, noradrenaline, and aldosterone immediately following surgery and 3 months postoperatively. ANP and BNP levels increased significantly over $24 \mathrm{~h}$ but returned to baseline at 3 months. Moreover, epicardial amputation led to significantly decreased BP at $24 \mathrm{~h}$ and 3 months postoperatively, but this effect was not observed with endocardial LAA devices. In addition, phenomena such as changes in fat breakdown, adipokines, free fatty acids, and glucose metabolism were observed with epicardial devices. The exact mechanisms for these have not been fully elucidated, and related studies have not been conducted. However, they seem to be regulated by LAA-secreted natriuretic peptide and autonomic innervation. Since both eventually achieve downregulation of the renin-angiotensin-aldosterone system, large-scale clinical observational and verification studies need to be conducted in the future. Meanwhile, BP reduction over the long term and alleviation of after-loading should contribute to the suppression of arteriosclerosis progression.

\section{Cases in Which LAA Procedures Are Effective}

Preoperative imaging tools such as heart CT and TEE are essential for identifying the LAA's anatomical structure and for the creation of a protocol, including the selection of the appropriate device. Anatomical items that need to be taken into consideration during preoperative imaging include the size of the LAA, its morphology (sharp corners in the chicken wing type, short necks of $<10 \mathrm{~mm}$ in the cauliflower type), and the presence of pectinate muscles, trabecula, lobation, and nodules. The presence of these structures can create difficulties when inserting the endocardial device, making it advisable to select an appropriate epicardial method.

It has recently been reported that it is possible to predict the risk of thrombosis onset within the LAA hemodynamically during sinus rhythm and AF by LAA morphological type. ${ }^{49)}$ If intra-LAA thrombi can be predicted preoperatively, it would be especially useful in selecting the device to be used in the procedure and the timing of the surgery.

Moreover, the use of a novel surgical device known as the AtriClip in LAA closure procedures has shown encouraging results. ${ }^{50)}$ This device may be applied to minimally invasive cardiac surgery (MICS) using small incisions and thoracoscopy. ${ }^{51)}$

\section{LAA Management at Juntendo}

From 2011 to 2017, 1018 cases of off-pump coronary artery bypass grafting (OPCAB) were performed at our hospital, and we conducted a comparison of exclusive OPCAB procedures and simultaneous LAA amputation procedures. 30\% of the patients developed POAF. Simultaneous LAA amputation during coronary bypass was 
low cost and safe, and the risk of cerebrovascular accident occurrence due to POAF was suppressed (earlyonset stroke: $0.9 \%$ in the OPCAB-only group vs. $0.4 \%$ in the LAA amputation group, late-onset stroke: 4.5 vs. $1.7 \%$, respectively). In the LAA amputation group, the frequency of arrhythmia such as new AF after surgery (both groups: $30 \%$ ) did not increase without the occurrence of adverse events, but detailed analysis of the changes such as that in BP following amputation and measurements of ANP and BNP are required. ${ }^{52)}$

\section{Future Perspectives}

Understanding LAA anatomy and physiology, and morphological changes, both size and functional, accompanying LAA remodeling during AF are essential. Since $\mathrm{AF}$ is an independent risk factor for cerebrovascular disorders throughout the recovery period post-openheart surgery, aggressive LAA management should be performed.

LAA closure reduces the risk of AF-related cerebrovascular accidents and is more cost-effective than anticoagulant therapy. Therefore, we expect a novel concept to arise involving new LAA management strategies with novel devices, MICS-LAA amputation, and LAA amputation procedures.

\section{Acknowledgment}

We would like to thank Editage (www.editage.com) for English language editing.

\section{Disclosure Statement}

The research did not receive any specific grant from any funding agency in the public, commercial, or notfor-profit sector.

The authors certify that there is no conflict of interest with any financial organization regarding the material discussed in the manuscript.

\section{References}

1) Saito $T$, Matsuyama $T$, Inoue $S$. The anatomical characteristics of the human atrium that are involved in atrial fibrillation. Jpn J Electrocardiology 2005; 25 suppl: 3-11. (in Japanese)

2) Anderson RH, Becker AE. The development of the heart. In: Anderson RH, Becker AE, eds.; Cardiac anatomy. London: Gower Medical Publishing, 1980, 10.1-30.

3) Kishima H, Mine T, Ashida K, et al Does left atrial appendage morphology influence left atrial appendage flow velocity? Circ J 2015; 79: 1706-11.

4) de Bold AJ, Bruneau BG, Kuroski de Bold ML. Mechanical and neuroendocrine regulation of the endocrine heart. Cardiovasc Res 1996; 31: 7-18.

5) Rodeheffer RJ, Naruse M, Atkinson JB, et al. Molecular forms of atrial natriuretic factor in normal and failing human myocardium. Circulation 1993; 88: 364-71.

6) Tabata T, Oki T, Yamada H, et al. Role of left atrial appendage in left atrial reservoir function as evaluated by left atrial appendage clamping during cardiac surgery. Am J Cardiol 1998; 81: 327-32.

7) Hoit BD, Shao Y, Tsai LM, et al. Altered left atrial compliance after atrial appendectomy. Influence on left atrial and ventricular filling. Circ Res 1993; 72: 167-75.

8) Vigna C, Russo A, De Rito V, et al. Frequency of left atrial thrombi by transesophageal echocardiography in idiopathic and in ischemic dilated cardiomyopathy. Am J Cardiol 1992; 70: 1500-1.

9) Fatkin D, Kelly RP, Feneley MP. Relations between left atrial appendage blood flow velocity, spontaneous echocardiographic contrast and thromboembolic risk in vivo. J Am Coll Cardiol 1994; 23: 961-9.

10) Pollick C, Taylor D. Assessment of left atrial appendage function by transesophageal echocardiography. Implications for the development of thrombus. Circulation 1991; 84: 223-31.

11) Nucifora G, Faletra FF, Regoli F, et al. Evaluation of the left atrial appendage with real-time 3-dimensional transesophageal echocardiography: implications for catheter-based left atrial appendage closure. Circ Cardiovasc Imaging 2011; 4: 514-23.

12) Stoddard MF, Dawkins PR, Prince CR, et al. Left atrial appendage thrombus is not uncommon in patients with acute atrial fibrillation and a recent embolic event: a transesophageal echocardiographic study. J Am Coll Cardiol 1995; 25: 452-9.

13) Allessie M, Ausma J, Schotten U. Electrical, contractile and structural remodeling during atrial fibrillation. Cardiovasc Res 2002; 54: 230-46.

14) Shirani J, Alaeddini J. Structural remodeling of the left atrial appendage in patients with chronic nonvalvular atrial fibrillation: Implications for thrombus formation, systemic embolism, and assessment by transesophageal echocardiography. Cardiovasc Pathol 2000; 9: 95-101.

15) Shinohara D, Matsushita $S$, Yamamoto T, et al. Reduction of c-kit positive cardiac stem cells in patients with atrial fibrillation. J Cardiol 2017; 69: 712-8.

16) Garabelli P, Stavrakis S, Kenney JFA, et al. Effect of 28-mm cryoballoon ablation on major atrial ganglionated plexi. JACC Clin Electrophysiol 2018; 4: 831-8. 
17) Hwang C, Chen PS. Ligament of Marshall: why it is important for atrial fibrillation ablation. Heart Rhythm 2009; 6: S35-40.

18) Shen MJ, Arora R, Jalife J. Atrial myopathy. JACC Basic Transl Sci 2019; 4: 640-54.

19) Go AS, Hylek EM, Phillips KA, et al. Prevalence of diagnosed atrial fibrillation in adults: national implications for rhythm management and stroke prevention: the AnTicoagulation and Risk Factors in Atrial Fibrillation (ATRIA) Study. JAMA 2001; 285: 2370-5.

20) Lloyd-Jones DM, Wang TJ, Leip EP, et al. Lifetime risk for development of atrial fibrillation: the Framingham Heart Study. Circulation 2004; 110: 1042-6.

21) Mozaffarian D, Benjamin EJ, Go AS, et al. Heart disease and stroke statistics-2016 update: a report from the American Heart Association. Circulation 2016; 133: e38-60.

22) Hamada R, Muto S. Simple risk model and score for predicting of incident atrial fibrillation in Japanese. J Cardiol 2019; 73: 65-72.

23) Li YG, Pastori D, Farcomeni A, et al. A simple clinical risk score (C2HEST) for predicting incident atrial fibrillation in Asian subjects: derivation in 471,446 Chinese subjects, with internal validation and external application in 451,199 Korean subjects. Chest 2019; 155: 510-8.

24) Endo D, Yamamoto T, Matsushita S, et al. Early postoperative atrial fibrillation after coronary artery bypass grafting aggravates late-onset atrial fibrillation? [Internet] EACTS 2020 [Cited October 9, 2020]. Available from: http://medialibrary.eacts.cyim.com/mediatheque $/$ media.aspx?mediaId=91005\&channel $=10233$.

25) Foulkes MA, Wolf PA, Price TR, et al. The stroke data bank: design, methods, and baseline characteristics. Stroke 1988; 19: 547-54.

26) Otite FO, Khandelwal $P$, Chaturvedi $S$, et al. Increasing atrial fibrillation prevalence in acute ischemic stroke and TIA. Neurology 2016; 87: 2034-42.

27) Gage BF, Waterman AD, Shannon W, et al. Validation of clinical classification schemes for predicting stroke: results from the National Registry of Atrial Fibrillation. JAMA 2001; 285: 2864-70.

28) Whitlock R, Healey JS, Connolly SJ, et al. Predictors of early and late stroke following cardiac surgery. CMAJ 2014; 186: 905-11.

29) Hart RG, Pearce LA, Aguilar MI. Meta-analysis: antithrombotic therapy to prevent stroke in patients who have nonvalvular atrial fibrillation. Ann Intern Med 2007; 146: 857-67.

30) Ruff CT, Giugliano RP, Braunwald E, et al. Comparison of the efficacy and safety of new oral anticoagulants with warfarin in patients with atrial fibrillation: a meta-analysis of randomised trials. Lancet 2014; 383: 955-62.

31) Kachroo S, Hamilton M, Liu X, et al. Oral anticoagulant discontinuation in patients with nonvalvular atrial fibrillation. Am J Manag Care 2016; 22: e1-8.
32) Yao X, Abraham NS, Alexander GC, et al. Effect of adherence to oral anticoagulants on risk of stroke and major bleeding among patients with atrial fibrillation. J Am Heart Assoc 2016; 5: e003074.

33) Badhwar V, Rankin JS, Damiano RJ, et al. The Society of Thoracic Surgeons 2017 clinical practice guidelines for the surgical treatment of atrial fibrillation. Ann Thorac Surg 2017; 103: 329-41.

34) Friedman DJ, Piccini JP, Wang T, et al. Association between left atrial appendage occlusion and readmission for thromboembolism among patients with atrial fibrillation undergoing concomitant cardiac surgery. JAMA 2018; 319: 365-74.

35) Holmes DR, Doshi SK, Kar S, et al. Left atrial appendage closure as an alternative to warfarin for stroke prevention in atrial fibrillation: a patientlevel meta-analysis. J Am Coll Cardiol 2015; 65: 2614-23.

36) Reddy VY, Akehurst RL, Armstrong SO, et al. Time to cost-effectiveness following stroke reduction strategies in AF: Warfarin versus NOACs versus LAA closure. J Am Coll Cardiol 2015; 66: 2728-39.

37) Aonuma K, Yamasaki H, Nakamura M, et al. Percutaneous WATCHMAN left atrial appendage closure for japanese patients with nonvalvular atrial fibrillation at increased risk of thromboembolism - first results from the SALUTE trial. Circ J 2018; 82: 2946-53.

38) Kefer J, Tzikas A, Freixa $X$, et al. Impact of chronic kidney disease on left atrial appendage occlusion for stroke prevention in patients with atrial fibrillation. Int J Cardiol 2016; 207: 335-40.

39) Piccini JP, Stevens SR, Chang Y, et al. Renal dysfunction as a predictor of stroke and systemic embolism in patients with nonvalvular atrial fibrillation: validation of the $\mathrm{R}(2)$ CHADS(2) index in the ROCKET AF (Rivaroxaban Once-daily, oral, direct factor Xa inhibition compared with vitamin $\mathrm{K}$ antagonism for prevention of stroke and embolism trial in atrial fibrillation) and ATRIA (AnTicoagulation and Risk factors In Atrial fibrillation) study cohorts. Circulation 2013; 127: 224-32.

40) Kubo S, Mizutani Y, Meemook K, et al. Incidence, characteristics, and clinical course of device-related thrombus after watchman left atrial appendage occlusion device implantation in atrial fibrillation patients. JACC Clin Electrophysiol 2017; 3: 1380-6.

41) Fauchier L, Cinaud A, Brigadeau F, et al. Devicerelated thrombosis after percutaneous left atrial appendage occlusion for atrial fibrillation. J Am Coll Cardiol 2018; 71: 1528-36.

42) Saw J, Fahmy P, DeJong P, et al. Cardiac CT angiography for device surveillance after endovascular left atrial appendage closure. Eur Heart J Cardiovasc Imaging 2015; 16: 1198-206.

43) Freixa X, Abualsaud A, Chan J, et al. Left atrial appendage occlusion: initial experience with the Amplatzer ${ }^{\mathrm{TM}}$ Amulet $^{\mathrm{TM}}$. Int J Cardiol 2014; 174: 492-6. 
44) Kanderian AS, Gillinov AM, Pettersson GB, et al. Success of surgical left atrial appendage closure: assessment by transesophageal echocardiography. J Am Coll Cardiol 2008; 52: 924-9.

45) Aryana A, Singh SK, Singh SM, et al. Association between incomplete surgical ligation of left atrial appendage and stroke and systemic embolization. Heart Rhythm 2015; 12: 1431-7.

46) Bajaj NS, Parashar A, Agarwal S, et al. Percutaneous left atrial appendage occlusion for stroke prophylaxis in nonvalvular atrial fibrillation: a systematic review and analysis of observational studies. JACC Cardiovasc Interv 2014; 7: 296-304.

47) Aminian A, Lalmand J, Tzikas A, et al. Embolization of left atrial appendage closure devices: A systematic review of cases reported with the watchman device and the amplatzer cardiac plug. Catheter Cardiovasc Interv 2015; 86: 128-35.

48) Lakkireddy D, Turagam M, Afzal MR, et al. Left atrial appendage closure and systemic homeostasis: the LAA HOMEOSTASIS study. J Am Coll Cardiol 2018; 71: 135-44.

49) Bosi GM, Cook A, Rai R, et al. Computational fluid dynamic analysis of the left atrial appendage to predict thrombosis risk. Front Cardiovasc Med 2018; 5: 34 .

50) Caliskan E, Sahin A, Yilmaz M, et al. Epicardial left atrial appendage AtriClip occlusion reduces the incidence of stroke in patients with atrial fibrillation undergoing cardiac surgery. Europace 2018; 20: e105-14.

51) Ellis CR, Aznaurov SG, Patel NJ, et al. Angiographic efficacy of the atriclip left atrial appendage exclusion device placed by minimally invasive thoracoscopic approach. JACC Clin Electrophysiol 2017; 3: 1356-65.

52) Endo D, Kato TS, Iwamura T, et al. The impact of surgical left atrial appendage amputation/ligation on stroke prevention in patients undergoing off-pump coronary artery bypass grafting. Heart Vessels 2017; 32: 726-34. 